\title{
A Strong Jet Signature in the Late-time Light Curve of GW170817
}

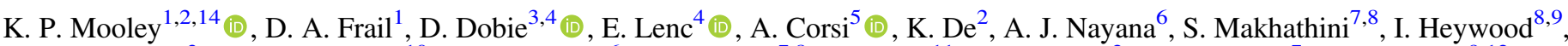

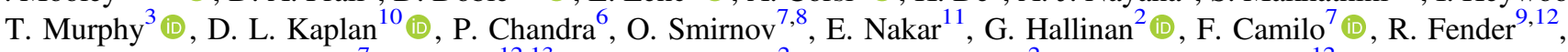 \\ S. Goedhart ${ }^{7}$, P. Groot ${ }^{12,13}$, M. M. Kasliwal ${ }^{2}$ (D), S. R. Kulkarni ${ }^{2}$ (D), and P. A. Woudt ${ }^{12}$ (iD \\ ${ }^{1}$ National Radio Astronomy Observatory, Socorro, NM 87801, USA; kunal@ astro.caltech.edu \\ ${ }^{2}$ Caltech, 1200 E. California Blvd. MC 249-17, Pasadena, CA 91125, USA \\ ${ }^{3}$ Sydney Institute for Astronomy, School of Physics, University of Sydney, Sydney, New South Wales 2006, Australia \\ ${ }^{4}$ ATNF, CSIRO Astronomy and Space Science, P.O. Box 76, Epping, New South Wales 1710, Australia \\ ${ }^{5}$ Department of Physics and Astronomy, Texas Tech University, Box 41051, Lubbock, TX 79409-1051, USA \\ ${ }^{6}$ National Centre for Radio Astrophysics, Tata Institute of Fundamental Research, Pune University Campus, Ganeshkhind Pune 411007, India \\ ${ }^{7}$ South African Radio Astronomy Observatory (SARAO), Pinelands 7405, South Africa \\ ${ }^{8}$ Department of Physics \& Electronics, Rhodes University, Grahamstown, 6139, South Africa \\ ${ }^{9}$ Denys Wilkinson Building, Keble Road, Oxford OX1 3RH, UK \\ ${ }^{10}$ Department of Physics, University of Wisconsin-Milwaukee, Milwaukee, WI 53201, USA
${ }^{11}$ The Raymond and Beverly Sackler School of Physics and Astronomy, Tel Aviv University, Tel Aviv 69978, Israel \\ ${ }_{12}$ Department of Astronomy, University of Cape Town, Private Bag X3, Rondebosch 7701, South Africa \\ ${ }^{13}$ Department of Astrophysics/IMAPP, Radboud University Nijmegen, P.O. Box 9010, 6500 GL Nijmegen, The Netherlands \\ Received 2018 August 30; revised 2018 October 17; accepted 2018 October 29; published 2018 November 16
}

\begin{abstract}
We present new $0.6-10 \mathrm{GHz}$ observations of the binary neutron star merger GW170817 covering the period up to 300 days post-merger, taken with the upgraded Karl G. Jansky Very Large Array, the Australia Telescope Compact Array, the Giant Metrewave Radio Telescope and the MeerKAT telescope. We use these data to precisely characterize the decay phase of the late-time radio light curve. We find that the temporal decay is consistent with a power-law slope of $t^{-2.2}$, and that the transition between the power-law rise and decay is relatively sharp. Such a slope cannot be produced by a quasi-isotropic (cocoon-dominated) outflow, but is instead the classic signature of a relativistic jet. This provides strong observational evidence that GW170817 produced a successful jet, and directly demonstrates the link between binary neutron star mergers and short-hard gamma-ray bursts. Using simple analytical arguments, we derive constraints on the geometry and the jet opening angle of GW170817. These results are consistent with those from our companion very long baseline interferometry paper, reporting superluminal motion in GW170817.
\end{abstract}

Key words: gravitational waves - radio continuum: stars - stars: neutron

\section{Introduction}

The detection of gravitational waves $(\mathrm{GW})$ from the binary neutron star merger event GW170817 (Abbott et al. 2017a) was accompanied by two distinct electromagnetic (EM) counterparts (Abbott et al. 2017b). The first EM counterpart was a short-lived, thermal-like component. It initially had bright optical/ultraviolet (UV) emission that faded on a timescale of a few days (Arcavi et al. 2017; Coulter et al. 2017; Evans et al. 2017; Lipunov et al. 2017; Soares-Santos et al. 2017; Valenti et al. 2017), to be replaced by redder emission that dominated the bolometric luminosity until it too faded on a timescale of a few weeks (Cowperthwaite et al. 2017; Drout et al. 2017; Kasliwal et al. 2017; Tanvir et al. 2017). Optical/near-infrared (near-IR) spectroscopy detected the spectral fingerprints of $r$-process elements (Pian et al. 2017; Smartt et al. 2017), and a broad consensus has formed that this thermal component was a "kilonova" powered by the dynamic ejecta from the merger event (e.g., Kasen et al. 2017).

Additionally, the EM counterpart was also detected as a prompt, low-luminosity burst of gamma-rays with duration $\sim 2 \mathrm{~s}$ but delayed from the GW merger event by 1.7 s (Goldstein et al. 2017). This was followed by the discovery of nonthermal "afterglow" emission at X-ray (Haggard et al. 2017; Margutti et al. 2017; Troja et al. 2017) and radio wavelengths

\footnotetext{
${ }^{14}$ Jansky Fellow (NRAO/Caltech).
}

(Hallinan et al. 2017), substantially delayed by 9 and 16 days, respectively. Both the prompt and the afterglow emission are thought to be generated in a relativistic shock, but a consensus on the nature of this second EM component has been slower to emerge. Two viable models were eliminated based on the early data. From the delayed onset of the afterglow it was conclusively shown that GW170817 was not a classical short-hard gammaray burst (SHB) with an on-axis jet (e.g., Kasliwal et al. 2017). Furthermore, an off-axis jet with a uniform or "top-hat" geometry was ruled out by the slow rise $\left(t^{+0.8}\right)$ of the radio emission up to 100 days post-merger (Mooley et al. 2018b), later confirmed at both optical and X-ray wavelengths (Lyman et al. 2018; Margutti et al. 2018; Ruan et al. 2018; Troja et al. 2018a).

There still remain two competing models for the prompt and afterglow emission of GW170817, both of which are well motivated physically. Both scenarios launch an ultra-relativistic jet (bulk Lorentz factor $\Gamma \sim 100$ ), pointing away from the Earth, that interacts with the neutron-rich material dynamically ejected during the merger to give rise to a mildly relativistic ( $\Gamma \sim 4$ ) outflow (a.k.a "cocoon") moving in the direction of the Earth. The mildly relativistic material is likely responsible for the gamma-ray signal (e.g., Kasliwal et al. 2017; Gottlieb et al. 2018; Matsumoto et al. 2018) and is primarily responsible for the slow rise $\left(t^{+0.8}\right)$ of the afterglow at early times (e.g., Mooley et al. 2018b). However, in one scenario the jet successfully escapes the dynamical ejecta, while in the other it fails to do so. In the literature the former case has been referred 
to as the successful jet-cocoon model or the structured jet model (where the successful jet is considered to be a narrow core with a "sheath" of lower Lorentz factor material), in which the jet dominates the late-time afterglow. The latter case is that of a choked jet, in which the afterglow is cocoon-dominated at all times. In the successful jet scenario, an observer located along the axis of the jet likely sees a regular SHB, while in the choked jet scenario they do not.

In the discussion that follows on the late-time light curves, we will refer to these as jet-dominated and cocoon-dominated outflows. Both outflow models require significant azimuthal and radial structure to explain the rise of the afterglow light curve (Nakar \& Piran 2018; Xie et al. 2018), but the open question that we hope to address here is whether or not the relativistic jet survived.

Several experimental tests have been suggested to distinguish between these two alternative scenarios (Gill \& Granot 2018; Lazzati et al. 2018; Nakar et al. 2018). Elsewhere we report on polarization measurements of GW170817 and our high angular resolution imaging (Corsi et al. 2018; Mooley et al. 2018a). In this Letter we focus on the continuum intensity of the afterglow light curve at late times. Several authors have noted that the rising portion of the light curve has limited discriminating power as the lower Lorentz factor ejecta dominate the emission in both models (D'Avanzo et al. 2018; Gill \& Granot 2018; Margutti et al. 2018; Nakar et al. 2018). However, as first noted by Dobie et al. (2018), and subsequently confirmed by Alexander et al. (2018), the afterglow light curve peaked around day 150 post-merger and has begun to decline. Our motivation for this work has been to characterize this decay phase (as attempted by previous studies that reported the peak and decline) and to see whether the geometry and dynamical state of the outflow can be inferred as it has been done in the past with the late-time light curves of long-duration gamma-ray burst (GRB) afterglows (Mészáros et al. 1998; Sari et al. 1999; Livio \& Waxman 2000).

In this Letter we present further radio observations of GW170817 using the Karl G. Jansky Very Large Array (VLA), the Australia Telescope Compact Array (ATCA), the upgraded Giant Metrewave Radio Telescope (uGMRT) and new observations from the MeerKAT telescope, covering the period 180-300 days post-merger. With this longer time-baseline we are able to accurately constrain the late-time power-law decay index and compare the results against expectations for a cocoon-dominated versus a jet-dominated outflow. In Section 2 we describe the observations and data reduction techniques employed, Section 3 gives the spectral and light curve analysis with the interpretation based on the widely used theory of GRB afterglows given in Section 4, and we end with the summary and conclusions in Section 5.

\section{Observations and Data Reduction}

Our observations of GW170817 carried out over the decline of the light curve are described below, and the observing log together with the flux densities of GW170817 are reported in Table 1. While our new VLA and ATCA observations span frequencies between 2 and $12 \mathrm{GHz}$, the addition of MeerKAT and uGMRT data gives us important spectral coverage below $2 \mathrm{GHz}$, which not only probes the low-frequency behavior of GW170817, but also gives very precise measurements of the radio spectral indices.
Table 1

Radio Observations of GW170817 during the Light Curve Decline

\begin{tabular}{lccccc}
\hline \hline UT Date & $\begin{array}{c}\Delta T^{\mathrm{a}} \\
(\text { days })\end{array}$ & Telescope & $\begin{array}{c}\nu \\
(\mathrm{GHz})\end{array}$ & $\begin{array}{c}F_{\nu} \\
(\mu \mathrm{Jy})\end{array}$ & $\begin{array}{c}\sigma_{\nu} \\
(\mu \mathrm{Jy})\end{array}$ \\
\hline 2018 Feb 16 & 183 & uGMRT & 0.65 & 211 & 34 \\
2018 Mar 2 & 197 & MeerKAT & 1.3 & 160 & 20 \\
2018 Mar 21 & 216 & VLA & 10 & 36.3 & 3.6 \\
2018 Mar 25-26 & 220 & VLA & 3 & 64.7 & 2.7 \\
2018 Mar 27 & 222 & ATCA & 7.25 & 39.7 & 7.2 \\
2018 Apr 26-May 5 & 257 & MeerKAT & 1.3 & 65.8 & 7.2 \\
2018 May 11-12 & 267 & VLA & 3 & 40.3 & 2.7 \\
2018 May 11 & 267 & ATCA & 7.25 & 25.0 & 4.1 \\
2018 May 13-25 & 275 & uGMRT $^{\mathrm{b}}$ & 0.65 & $<153$ & $\ldots$ \\
2018 Jun 7 & 294 & VLA & 3 & 31.2 & 3.6 \\
2018 Jun 11 & 298 & ATCA $^{\text {b }}$ & 7.25 & 23.4 & 4.2 \\
\hline
\end{tabular}

Notes.

${ }^{a}$ Mean epoch, days post-merger.

b The ATCA flux densities of GW170817 have a correction factor of 1.25 applied (i.e., the values have been decreased by 25\%; see Section 3.1 for details).

\subsection{VLA}

We observed GW170817 on 2018 March 21, March 25-26, May 11-12, and June 7 with the VLA (P.I.: A. Corsi). The Wideband Interferometric Digital Architecture correlator was used at $S$-band (2-4 GHz) and $X$-band $(8-12 \mathrm{GHz})$ to maximize sensitivity. We used PKS J1248-1959 as the phase calibrator and 3C286 as the flux density and bandpass calibrator. The data were calibrated and flagged for radio frequency interference (RFI) using the NRAO CASA (McMullin et al. 2007) pipeline. We then split and imaged the target data using the CASA tasks split and clean.

\section{2. $A T C A$}

We observed GW170817 with the ATCA (P.I.: D. Dobie and E. Troja) at three epochs between 2018 March to June. We determined the flux scale and bandpass response for all epochs using the ATCA primary calibrator PKS B1934-638. Observations of PKS B1245-197 were used to calibrate the complex gains. All observations used two bands of $2048 \mathrm{MHz}$ centered at 5.5 and $9.0 \mathrm{GHz}$.

We reduced the visibility data using standard MIRIAD (Sault et al. 1995) routines. The calibrated visibility data from both bands were combined, averaged to $32 \mathrm{MHz}$ channels, and imported into DIFMAP (Shepherd 1997). Bright field sources were modeled separately for each band using the visibility data and a combination of point-source and Gaussian components with power-law spectra. After subtracting the modeled field sources from the visibility data, GW170817 dominates the residual image. Restored naturally weighted images for each band were generated by convolving the restoring beam and modeled components, adding the residual map and averaging to form a wide-band image. Image-based Gaussian fitting with unconstrained flux density and source position was performed in the region near GW170817.

\section{3. $u G M R T$}

We observed GW170817 with the uGMRT in Band 3 (effective bandwidth 550-750 MHz) (PI: Mooley). The observations were carried out with $400 \mathrm{MHz}$ correlator bandwidth 
centered at $750 \mathrm{MHz}$ using the non-polar continuum interferometric mode of the GMRT Wideband Backend (Reddy et al. 2017). The epochs from 2018 May and 2018 June were divided into several short $(\sim 1-3 \mathrm{hr})$ observations carried out over several days. 3C286 was used as the absolute flux scale and bandpass calibrators, while phase calibration was done with 3C283. These data were calibrated and RFI flagged using a custom-developed pipeline in CASA. The data were then imaged interactively with the CASA task CLEAN, while incorporating a few iterations of phase-only self-calibration and one amplitude+phase self-calibration step.

\subsection{MeerKAT}

We observed GW170817 with the new MeerKAT telescope (Jonas \& MeerKAT Team 2016; Camilo et al. 2018) on 2018 March 02, April 26 and May 05. The first of these observations used 16 antennas and the ROACH2 correlator; the latter used 61 antennas and the SKARAB correlator. All observations were made at $L$-band, covering $900-1670 \mathrm{MHz}$. After flagging for RFI, the effective bandwidth used was $486 \mathrm{MHz}$. PKS 1934 -638 was used as the flux and bandpass calibrator and for initial phase reference. Data processing was done with the MeerKATHI pipeline (S. Makhatini et al. 2018, in preparation). To correct for the uncertainties in the frequency-dependent primary beam correction and pointing errors, we used direction-dependent gain calibration for bright sources spread across the $\sim 1^{\circ}$ field of view.

\section{Results}

\subsection{Spectral Analysis}

In Dobie et al. (2018) we studied the radio spectral evolution of GW170817, finding that over the first 120 days the radio spectral index was constant with a value of $\beta=-0.57 \pm 0.04$. This value of $\beta$ is fully consistent with the value derived by Alexander et al. (2018), $\beta=-0.74 \pm 0.2$, at 217 days. Here and elsewhere in this Letter we characterize the flux density evolution of the light curve using $F_{\nu}(t, \nu) \propto t^{\alpha} \nu^{\beta}$, where $\alpha$ and $\beta$ are the temporal and spectral power-law indices, respectively.

We have used the new data to look for changes in the radio spectral index. Specifically, we have searched for a steepening of the spectral index of order $\Delta \beta=0.5$ as expected if the synchrotron cooling break had moved through the radio band on timescales of 200-300 days (Sari et al. 1998). We compare our radio measurements from 2018 May reported in Table 1 with the X-ray measurement from 2018 May 3-5 (Alexander et al. 2018; Nynka et al. 2018; Troja et al. 2018c) to derive a spectral index of $\beta_{\mathrm{XR}}=-0.56 \pm 0.01$. Our most precise (simultaneous) two-point measurement of the radio-only spectral index is with the VLA data between 3 and $10 \mathrm{GHz}$ in 2018 March (day 220), $\beta=-0.52 \pm 0.09$. Both these measurements are consistent with the precise radio-to-X-ray spectral index $\beta=-0.584 \pm 0.006$ at 160 days post-merger derived by Margutti et al. (2018). We see no evidence for a spectral steepening, and can rule out the presence of a cooling break for which we expect $\beta \sim-1.1$ (Alexander et al. 2018).

In these spectral comparisons between telescopes, we see some evidence for a scaling offset in the late-time ATCA data. While the in-band 5.5-9 GHz spectral indices derived for the ATCA data are consistent with -0.584 broadband value above, the flux densities are higher by about $25 \%$ when the data are compared with contemporaneous measurements made with the

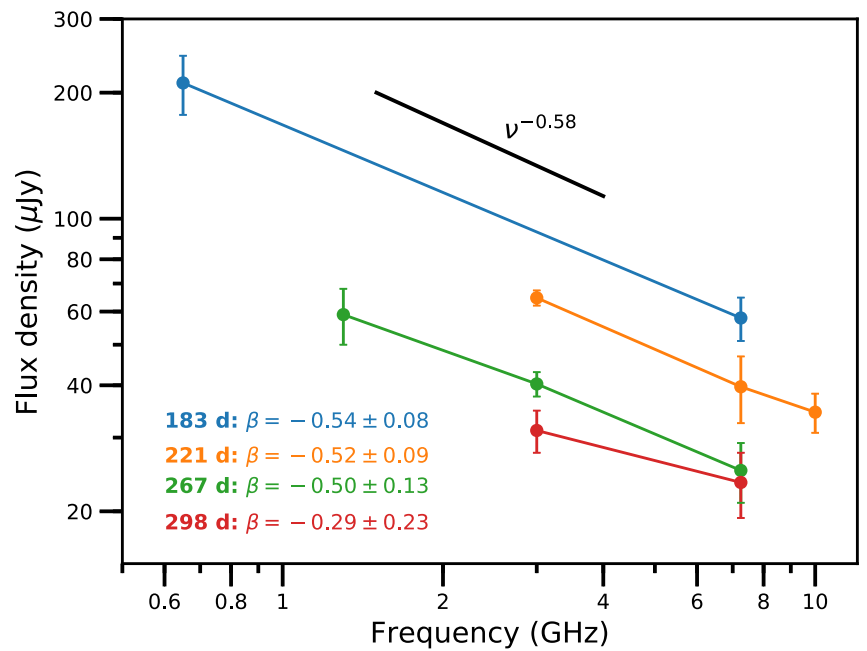

Figure 1. Radio spectral indices between 0.6 and $10 \mathrm{GHz}$ spanning four epochs observed during the decline part of the light curve. The different epochs are color coded. The approximate number of days post-merger and the corresponding spectral indices are given in the legend. A joint fit to all the radio data implies $\beta=-0.53 \pm 0.04$ (see Section 3.2), which is in good agreement with the radio to X-ray spectral index measurements (shown by the black $\nu^{-0.58}$ line). With this measurement, we can rule out any spectral steepening expected, for example, due to the presence of a cooling break in or between the radio/X-ray bands.

VLA, MeerKAT and uGMRT. The origin of excess in the ATCA data is currently being investigated. ${ }^{15}$ In Table 1 we have reported the corrected ATCA flux densities. The radioonly spectral index measurements from four epochs observed during the decline of the light curve are shown in Figure 1. The spectral index between the $0.65 \mathrm{GHz}$ uGMRT and $7.25 \mathrm{GHz}$ ATCA data (corrected from the value reported in Dobie et al. 2018) obtained around 2018 February 16 (183 days postmerger) is $-0.54 \pm 0.08$. Below, we describe a joint fit to multi-frequency light curve data, including the spectral and temporal indices.

\subsection{Light Curve Analysis}

We began by performing a joint fit to radio data published until day 300 post-merger. This includes the data in Table 1 together with Hallinan et al. (2017), Alexander et al. (2017), Mooley et al. (2018b), Margutti et al. (2018), and Dobie et al. (2018). We used a smoothly broken power-law model, incorporating the frequency dependence, of the form $2^{1 / s} \nu^{\beta} F_{\nu, p}\left(t^{-s \alpha_{1}}+t^{-s \alpha_{2}}\right)^{-1 / s}$ (Beuermann et al. 1999; Alexander et al. 2018). Here, $\nu$ is the observing frequency normalized to $3 \mathrm{GHz}, F_{\nu, p}$ is the flux density at $3 \mathrm{GHz}$ at the time of light curve peak, $t$ is the time in units of the time to light curve peak $\left(t_{p}\right), s$ is the smoothness parameter, and $\alpha_{1}, \alpha_{2}$ are the power-law rise and decay slopes. This Markov chain Monte Carlo (MCMC) fitting was done ${ }^{16}$ using the Python package emcee (Foreman-Mackey et al. 2013). We obtain best-fit values of $F_{\nu, p}=118_{-7}^{+14} \mu \mathrm{Jy}, t_{p}=167_{-7}^{+14}$ days, $\alpha_{1}=0.80_{-0.05}^{+0.06}$, $\alpha_{2}=-2.16_{-0.61}^{+0.23}, \quad \log _{10}(s)=0.59_{-0.37}^{+0.77}$ and $\beta=-0.61_{-0.07}^{+0.03}$ $(68 \%$ confidence interval, i.e., $1 \sigma$; see Table 2$)$. We also introduced a scale factor into the MCMC fit to explore a

\footnotetext{
15 This appears to be a systematic offset. The origin of the offset is not in the flux scale, as the radio spectrum of the phase reference source is consistent between all of the epochs from the different telescopes. The ATCA array has a more compact configuration, so it may be due to host galaxy contamination.

${ }^{16}$ We chose 100 walkers, 1000 steps, and flat priors on all of the parameters.
} 
Table 2

Parameters Obtained from Fitting a Smoothly Broken Power-law Model to the Radio Light Curve

\begin{tabular}{|c|c|c|c|c|c|c|}
\hline No. & $\begin{array}{c}F_{\nu, p} \\
(\mu \mathrm{Jy})\end{array}$ & $\begin{array}{c}t_{p} \\
\text { (days) }\end{array}$ & $\alpha_{1}$ & $\alpha_{2}$ & $\log _{10}(\mathrm{~s})$ & $\beta$ \\
\hline 1 & $118_{-7}^{+14}$ & $167_{-7}^{+14}$ & $0.80_{-0.05}^{+0.06}$ & $-2.16_{-0.61}^{+0.23}$ & $0.59_{-0.37}^{+0.77}$ & $-0.61_{-0.07}^{+0.03}$ \\
\hline 2 & $98_{-9}^{+8}$ & $174_{-6}^{+9}$ & $0.78 \pm 0.05$ & $-2.41_{-0.42}^{+0.26}$ & $0.70_{-0.34}^{+0.49}$ & $-0.53 \pm 0.04$ \\
\hline $3^{*}$ & $120 \pm 9$ & $164 \pm 7$ & $0.83 \pm 0.07$ & $-2.23 \pm 0.24$ & $\ldots$ & $\ldots$ \\
\hline
\end{tabular}

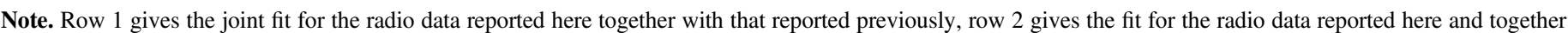

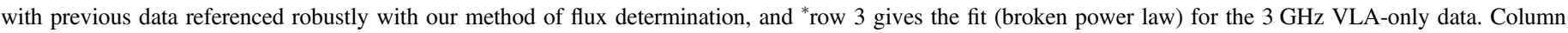

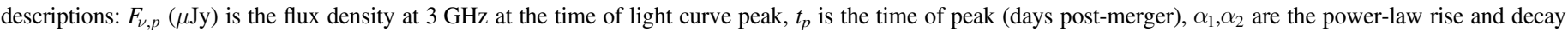
slopes, $s$ is the smoothness parameter, and $\beta$ is the spectral index. See Section 3.2 for details.

possible $25 \%$ offset in the ATCA flux densities suggested by the spectral fits in Section 3.1. We find that a scaling factor of $\sim 20 \%$ is slightly preferred over unity. ${ }^{17}$

Next we fit only the data in Table 1 together with previous data at $0.65,1.5,3$, and $7.25 \mathrm{GHz}$ referenced robustly with our method of flux determination (Hallinan et al. 2017; Dobie et al. 2018; Mooley et al. 2018b, flux density values given in Table 3). Our best-fit values are given in Table 2, and are consistent with the fit using all of the data above. In particular, we find $\alpha_{2}=-2.4_{-0.4}^{+0.3}$. Figure 2 shows the multi-frequency radio data scaled to $3 \mathrm{GHz}$, and the joint fit to these data (solid line). Figure 3 shows the corner plot with the results of the MCMC fit.

By taking the limit in which the $t^{-s \alpha_{1}}$ term dominates ${ }^{18}$ over the $t^{-s \alpha_{2}}$ term in the smoothly broken power-law expression given above, we derive that the transition from the power-law rise to the power-law decay takes place between $158_{-18}^{+13}$ and $183_{-15}^{+42}$ days post-merger, i.e., over a timescale of $24_{-24}^{+58}$ days. This implies that the transition from $\alpha_{1}$ to $\alpha_{2}$ is fairly sharp, possibly taking place over a small fraction of the time taken to reach the light curve peak. We return to this point in Section 4.

The reduction in the uncertainties for $\alpha_{2}$ in the second fit hints that there may still be systematic uncertainties involved in the calibration across data taken from different telescopes and obtained at different frequencies. Thus we chose to independently fit the $3 \mathrm{GHz}$ VLA-only data as was first done in (Mooley et al. 2018a). In this case, the light curve is too sparsely sampled to be able to fit for the smoothness parameter, and hence we use a simple broken power-law model (this corresponds to $s \rightarrow \infty$ ) instead. Table 2 gives the parameter values from the fitting, and we find that $\alpha_{2}=-2.2 \pm 0.2$. The decline is somewhat shallower than, but in good agreement with, the smoothly broken power-law model parameters. The remaining parameters such as the slope of the rise, the peak flux density, and the time of peak all agree well with each other and with previous fits in the literature. The main point here is that our key results are robust to different choices of the data that we used in the fit.

Summarizing, we measure a sharp transition of the afterglow light curve of GW170817 about 170 days post-merger with a steep power-law slope of $\alpha_{2}=-2.2$. The result confirms our

\footnotetext{
17 Median flux multiplication factor is 0.83 and the $68 \%$ confidence interval is 0.75-1.07. Note that the scaling factor is required for all ATCA data (reported here and previously). As an experiment, we have also performed a fit without including an ATCA flux scaling factor in the MCMC analysis, and the $\chi^{2}$ is significantly worse in this case as expected (87.4 versus 67.4$)$. Nevertheless, we get $\alpha_{2}=1.86_{-0.23}^{+0.17}$ without the scaling factor.

18 We derive the time at which one term dominates over the other by a factor of $\sim 20$. The quoted time values are the median of the distributions and their 16 and 84 percentiles are quoted as the uncertainties.
}

Table 3

Previous Radio Observations of GW170817

\begin{tabular}{lccccc}
\hline \hline UT Date & $\begin{array}{c}\Delta T^{\mathrm{a}} \\
(\text { days })\end{array}$ & Telescope & $\begin{array}{c}\nu \\
(\mathrm{GHz})\end{array}$ & $\begin{array}{c}F_{\nu} \\
(\mu \mathrm{Jy})\end{array}$ & $\begin{array}{c}\sigma_{\nu} \\
(\mu \mathrm{Jy})\end{array}$ \\
\hline 2017 Sep 2-4 & 17.4 & VLA & 3 & 15.4 & 2.5 \\
2017 Sep 8 & 22.36 & VLA & 3 & 22.5 & 3.4 \\
2017 Sep 10 & 24.26 & VLA & 3 & 25.6 & 2.9 \\
2017 Sep 17 & 31.33 & VLA & 3 & 34.0 & 3.6 \\
2017 Oct 2 & 46.25 & VLA & 3 & 44 & 4 \\
2017 Oct 10 & 54.29 & VLA & 3 & 48 & 6 \\
2017 Oct 13 & 57.19 & VLA & 3 & 61 & 9 \\
2017 Nov 18 & 93.13 & VLA & 3 & 70 & 6 \\
\hline 2017 Sep 5 & 18.66 & ATCA & 7.25 & 20.0 & 4.8 \\
2017 Nov 1 & 75.49 & ATCA & 7.25 & 35.9 & 4.3 \\
2017 Nov 17 & 92.4 & ATCA & 7.25 & 31.7 & 5.6 \\
2017 Dec 2 & 107.36 & ATCA & 7.25 & 53.2 & 4.5 \\
2017 Dec 20 & 125.3 & ATCA & 7.25 & 58.2 & 5.0 \\
2018 Jan 13 & 149.26 & ATCA & 7.25 & 60.6 & 4.3 \\
2018 Feb 1 & 181.64 & ATCA & 7.25 & 57.9 & 6.9 \\
\hline 2017 Oct 21 & 65.14 & GMRT & 0.61 & 117 & 42 \\
\hline
\end{tabular}

Notes. 1. Compilation from: Hallinan et al. (2017), Mooley et al. (2018b), Dobie et al. (2018). 2. The ATCA flux densities of GW170817 have a correction factor of 1.25 applied (see Section 3.1 for details).

${ }^{a}$ Mean epoch, days post-merger.

earlier determination of $\alpha_{2}$ first reported in Mooley et al. (2018a). With less data and a shorter time-baseline Dobie et al. (2018) derive a more shallow decay index $\alpha_{2}=-1.6 \pm 0.2$, which is similar to the value that Alexander et al. (2018) find, $\alpha_{2}=-1.6_{-0.3}^{+0.2}$. Our more precise values of $\alpha_{2}$ lie within the $68 \%$ confidence interval of Troja et al. (2018c) but we measure a larger value for the smoothness parameter.

\section{Discussion}

Before interpreting the light curve of GW170817 directly, it is illustrative to review the two asymptotes of late-time light curve behavior from afterglow models. Afterglow spectra and the light curves of GRBs have long been used to infer the geometry and dynamical state of the ejecta (e.g., Galama et al. 1998; Harrison et al. 1999). For a spherical relativistic fireball the flux density will decline as $F_{\nu} \propto t^{\alpha} \nu^{\beta}$, in which $\alpha=-3(p-1) / 4$ and $\beta=-(p-1) / 2$ when the observing frequency $\nu_{\mathrm{o}}$ is below the synchrotron cooling break $\nu_{c}$, and $\alpha=-(3 p-2) / 4$ and $\beta=-p / 2$ when the cooling break lies below the observing frequency (Sari et al. 1998). Here $p$ is the usual power-law index for the energy of the accelerated electrons $(p>2)$. For a jet viewed on-axis at late times the 


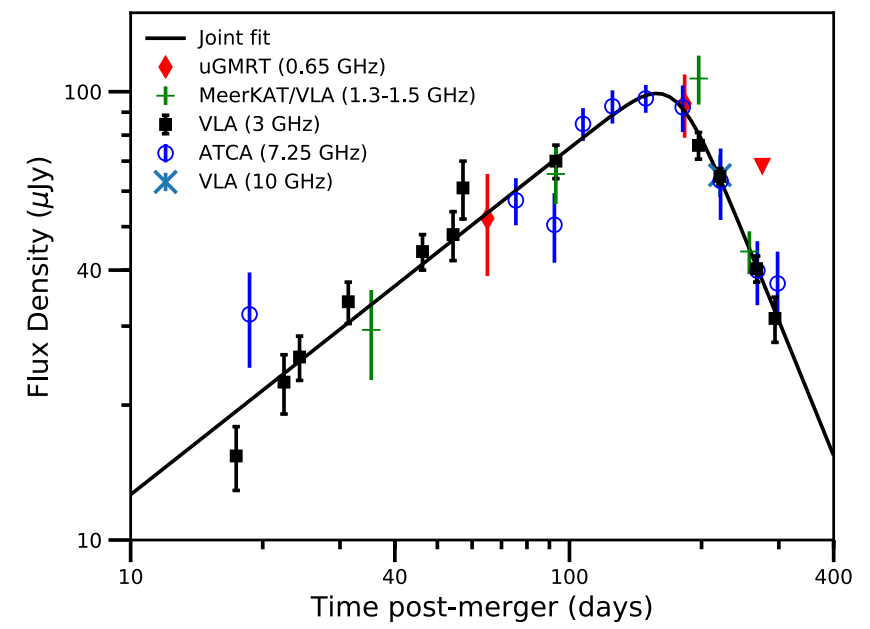

Figure 2. Radio light curve of GW170817 spanning multiple frequencies, and scaled to $3 \mathrm{GHz}$ using the spectral index $\left(\nu^{-0.53}\right)$ derived from our MCMC analysis. The data from the VLA (filled black squares for $3 \mathrm{GHz}$ and green crosses for $1.5 \mathrm{GHz}$ ), the ATCA (blue circles), the MeerKAT (green crosses) and the uGMRT (red diamonds for detections and triangle for upper limit) are as reported in Table 1 . We also include the data at $0.65,1.5,3$, and $7.25 \mathrm{GHz}$ reported previously (Hallinan et al. 2017; Dobie et al. 2018; Mooley et al. $2018 \mathrm{~b}$ ). Our best-fit smoothed broken power-law model to all these data (see Section 3.2) is shown as a solid curve. The power-law decline index obtained is $-2.4_{-0.4}^{+0.3}$. For comparison, a broken power-law fit to the $3 \mathrm{GHz}$ VLA-only data gives $-2.2 \pm 0.2$. Both fits are thus consistent with $t^{-p}$ decline in the light curve, where $p$ is the electron power-law distribution index.

power-law decay index is $\alpha=-p$, independent of whether the cooling break is above (i.e., $\beta=-(p-1) / 2$ ) or below (i.e., $\beta=-p / 2$ ) the observing frequency (Sari et al. 1999).

For GW170817 we show here (Section 3.1), as also demonstrated elsewhere (Dobie et al. 2018; Alexander et al. 2018), that in the radio regime $\beta$ is consistent with the value found from fitting across the radio and $\mathrm{X}$-ray regimes, $\beta=-0.584$, and that $\nu_{c}$ lies well above the radio (and likely also the X-ray) band. Thus $\beta=-(p-1) / 2$ and $p=2.17$, for which we expect the late-time power-law decay index $\alpha$ to lie between -0.88 (i.e., quasi-spherical, cocoon-dominated) and -2.17 (jet-dominated). Eventually we also expect the outflow to become non-relativistic, and this can give rise to an achromatic change in the GRB afterglow light curves. Dynamical transitions to the non-relativistic phase have been claimed for both spherical and jet-like outflows (Frail et al. 2000, 2005; van der Horst et al. 2008). The timescale on which this occurs is approximately when the rest mass energy of the material swept up by the shock is comparable to the kinetic energy of the outflow (Frail et al. 2000). The sideways expansion of the jet becomes important and eventually the outflow becomes quasi-spherical (Frail et al. 2000). At this time $\alpha_{\mathrm{nr}}=-(15 p-21) / 10$ for $\nu_{\circ}<\nu_{c}$ and $\alpha_{\mathrm{nr}}=-(3 p-4) / 2$ for $\nu_{\circ}>\nu_{c}$ (Livio \& Waxman 2000). Thus for the nominal parameters of GW170817, a spherical outflow undergoing a non-relativistic transition would be expected to show an achromatic steepening, while for a jet the light curve would flatten, both with a value of $\alpha_{\mathrm{nr}}=-1.15$.

The afterglow light curves of jet-like outflows are altered by observing them at different viewing angles away from their symmetry axis. In this case the structure of the ejecta becomes important. Early work investigated the role of viewing angle for simple uniform or top-hat jets (Livio \& Waxman 2000) and jets with azimuthal structure (e.g., Rossi et al. 2002; Kumar \& Granot 2003). More recent modeling has considered structure jets whose ejecta have both azimuthal and radial structure (Gottlieb et al. 2018; Kathirgamaraju et al. 2018; Lazzati et al. 2018; Xie et al. 2018). The effects of jet structure and viewing angle are most pronounced at or near the peak of the light curve. These models generally predict a slow temporal evolution of $\alpha$, with the break between the rise and the decay taking place over a significant fraction of the peak time (Granot \& Kumar 2003; Panaitescu \& Kumar 2003; Gill \& Granot 2018; Lamb et al. 2018). However, at late times all of these off-axis light curves models approach the behavior of an onaxis jet where the slope of the temporal index, as noted earlier, is $\alpha=-p$.

While the predicted late-time light curves of afterglows exhibit a diverse range of behaviors, the observed decay of GW170817 is remarkably simple. A single power law with $\alpha_{2}=-2.4_{-0.4}^{+0.3}(2.2 \pm 0.2$ for the $3 \mathrm{GHz}$ VLA-only data) fits all of the data post-peak. This power-law index is a clear signature of a relativistic jet. This is a strong jet-dominated outflow; i.e., there is no support for intermediate slopes as might be expected if a quasi-spherical cocoon was contributing to the emission. Likewise, we see no evidence for a spectral change due to synchrotron cooling (Section 3.1), nor do we see a dynamical transition to non-relativistic motion that would manifest itself by an achromatic break in the light curve. Another important feature of the light curve in Figure 2 is the sharpness of the transition from a power-law rise to decay. The change from $t^{0.8}$ to $t^{-p}$ takes place over $24_{-24}^{+58}$ days, a result that appears to be at odds with the predicted temporal evolution of $\alpha$ for current offaxis, structured jet models (Granot \& Kumar 2003; Panaitescu \& Kumar 2003; Gill \& Granot 2018). More detailed modeling of GW170817 is needed to see whether or not structured jetlike outflows can reproduce this sharp transition.

The sharpness of the peak and the slope of the power-law decline depend on the viewing/observing angle, $\theta_{v}$, and jet core half-opening angle, ${ }^{19} \theta_{j}$, and more specifically on the ratio between them. In order to constrain this ratio we consider only the contribution from the core of the jet, which dominates the emission near the peak and during the decay. Thus, while the rising part of the light curves that we calculate does not fit the observations, the peak and the decay should. Using this approximation, we can now derive constraints on $\theta_{j} / \theta_{v}$ that provides the observed transition from the peak of the light curve and the steep decline.

We make a rough analytic approximation. The peak occurs approximately when we start seeing the near edge of the jet core, and the $t^{-p}$ power-law decline begins roughly when the jet centroid comes into view. The sharpness of the light curve peak and the immediate transition to $t^{-p}$ decline implies that we are in the regime ${ }^{20} \theta_{v}-\theta_{j} \gg \theta_{j}$. We denote by $t_{1}$ as the time that we see the edge of the jet, namely $\Gamma\left(t_{1}\right) \simeq 1 /\left(\theta_{v}-\theta_{j}\right)$, and $t_{2}$ as the time that we see the jet axis, $\Gamma\left(t_{2}\right) \simeq 1 / \theta_{v}$. Now, ignoring sideways spreading of the jet we can approximate $\Gamma \propto t^{-3 / 8}$ (e.g., Sari et al. 1998) to obtain $\Delta t / t=\left(t_{2}-t_{1}\right) / t_{2} \simeq\left[\theta_{v}^{8 / 3}-\left(\theta_{v}-\theta_{j}\right)^{8 / 3}\right] / \theta_{v}^{8 / 3} \simeq(8 / 3) \theta_{j} / \theta_{v}$. Here we use the approximation that $\theta_{j} / \theta_{v}$ is much smaller than unity. Observationally, $t_{1}$ occurs sometime during the transition from the $t^{0.8}$ rise to the peak of the light curve and $t_{2}$ occurs

\footnotetext{
19 The energy distribution at the core is expected to be roughly uniform, so we approximate its contribution as being generated by a top-hat jet with halfopening angle, $\theta_{j}$.

${ }^{20}$ If $\left(\theta_{v}-\theta_{j}\right) \lesssim \theta_{j}$ there will be a long-lived phase during which the light curve decays as $t^{-1}$
} 


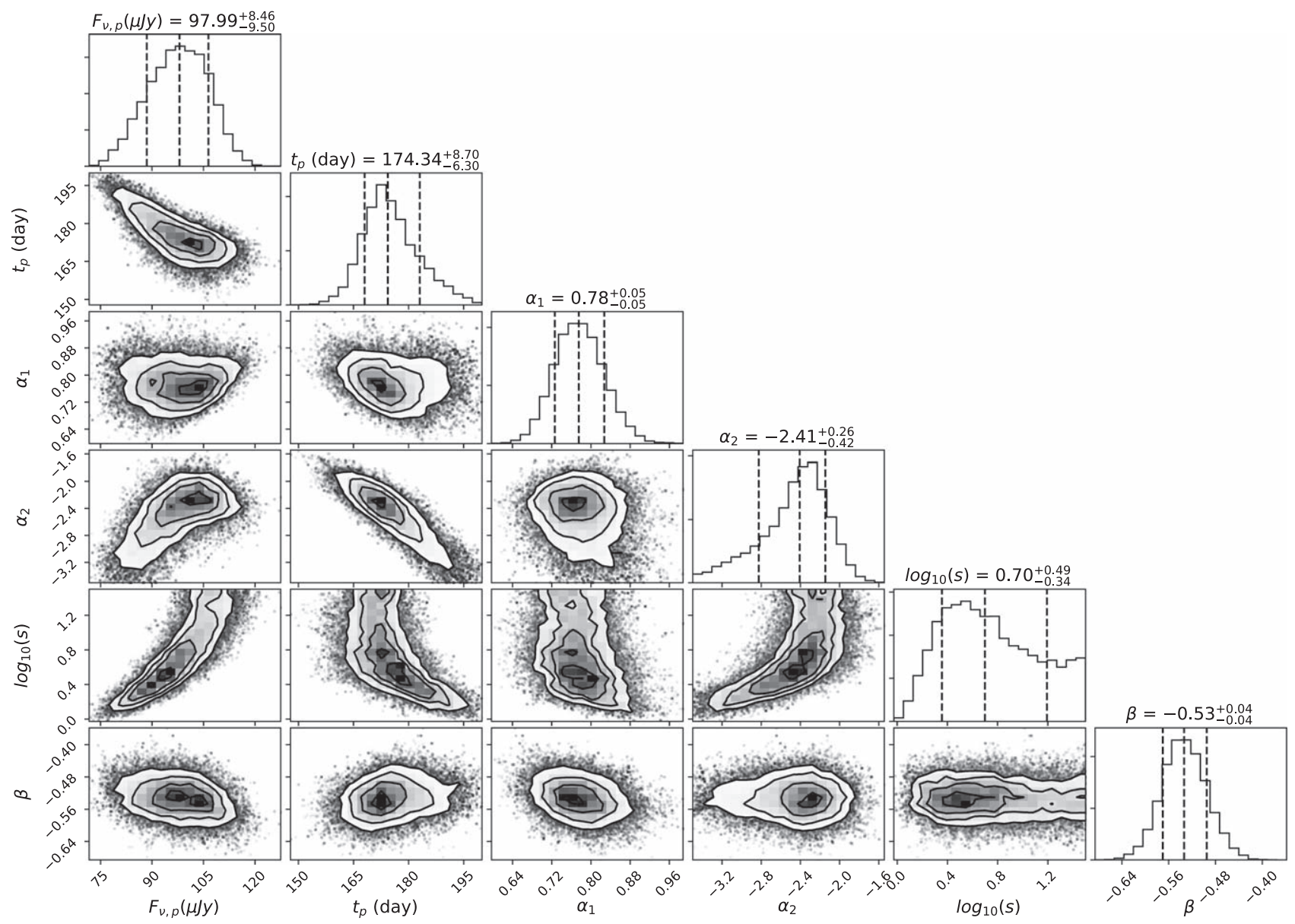

Figure 3. Corner plot showing the results of our MCMC fitting of the radio light curve (Figure 2) using the VLA, ATCA, MeerKAT, and uGMRT data. $F_{\nu, p}$ is the flux density at $3 \mathrm{GHz}$ at the time of light curve peak, $t_{p}$ is the time to light curve peak, $\alpha_{1}, \alpha_{2}$ are the power-law rise and decay slopes, $s$ is the smoothness parameter, and $\beta$ is the spectral index. See Section 3.2 for details. In each contour plot and histogram, the 16, 50, and 84 percentiles are marked.

when the decay phase approaches $t^{-p}$. Using the results from our MCMC analysis, we find that $\Delta t / t \lesssim 1 / 3(68 \%$ confidence or better, depending on the where $t_{1}$ and $t_{2}$ lie), indicating that $\theta_{v} \gtrsim 8 \theta_{j}$. To verify this simple estimate we produced light curves from a top-hat jet using semi-analytic code. ${ }^{21}$ We find that light curves that fit the transition seen from the peak to the decline of the light curve have $\theta_{v} \simeq 6 \theta_{j}$. Together with the upper limit on the observing angle from the Laser Interferometer Gravitational-Wave Observatory (LIGO)-Virgo Collaboration $\left(\theta_{v} \lesssim 28^{\circ}\right.$; Abbott et al. 2017a), our constraint implies $\theta_{j} \lesssim 5^{\circ}$. If instead we use the estimate of $\Gamma \simeq$ $4.1 \pm 0.5$ close to the peak of the light curve from the very long baseline interferometry (VLBI) measurement (Mooley et al. 2018a), then we get $\theta_{j} \lesssim 3^{\circ}$ and $\theta_{v} \simeq 15^{\circ}$.

\section{Summary and Conclusions}

In this companion Letter to our polarization and high angular resolution imaging studies, we have presented deep VLA, ATCA, MeerKAT, and uGMRT observations (Section 2) of GW170817 post-peak of the light curve, i.e., between 180 and

\footnotetext{
21 The semi-analytic code (Soderberg et al. 2006) takes proper account of all relativistic effects such as Lorentz boost and light arrival time from each point in the jet and it includes lateral spreading of the jet. Following corollary comparison of the code results to BOXFit (van Eerten et al. 2012), we use lateral spreading of $30 \%$ of the local thermal speed. We verify that varying the spread velocity, including taking no spreading at all, does not change the result significantly.
}

300 days post-merger. Our spectral analysis does not yield any evidence for the cooling break to have entered the radio band, or of any achromatic steepening in the light curve indicating the transition of the outflow into the non-relativistic regime (Section 3.1). We find that the light curve decay is consistent with $t^{-2.2}$ (Section 3.2), which is a classic signature of a jet where the slope of the decay is equal to the power-law energy index $p$ of the synchrotron-emitting electrons. We also find that the transition from the power-law rise to decay $\left(t^{0.8}\right.$ to $\left.t^{-2.2}\right)$ is fairly sharp.

The data on the light curve decline reported previously (Alexander et al. 2018; Dobie et al. 2018) has not been sufficiently constraining to unambiguously distinguish between cocoon-dominated and jet-dominated emission. The new data that we have reported here securely implies a decline in the light curve consistent with $t^{-p}$ (where $p$ is the electron energy distribution power-law index), which is a strong evidence for the late-time afterglow being jet-dominated. Our observations support recent claims from hydrodynamic modeling that GW170817 produced a successful jet (Duffell et al. 2018). Using these new data we can also derive robust constraints on the smoothness parameter $(s)$ and therefore the sharpness of the light curve peak, something that has not been possible with previously reported data. Together with the sharpness of the peak, the steep decline indicates that the jet is extremely narrow and that most of the outflow energy of GW170817 resides in the jet. Through simple analytical arguments we are able to 
place a constraint on the geometry, $\theta_{v} \gtrsim 8 \theta_{j}\left(\theta_{v} \gtrsim 6 \theta_{j}\right.$ with semi-analytical modeling; $\theta_{v}$ is the viewing angle and $\theta_{j}$ is the jet half-opening angle), and implies $\theta_{j} \lesssim 5^{\circ}$ if we further use the viewing angle constraint provided by the LIGO-Virgo Collaboration. Using $\Gamma \simeq 4$ close to the peak of the light curve (estimated from the observed superluminal motion in GW170817) gives $\theta_{j} \lesssim 3^{\circ}$ and $\theta_{v} \simeq 15^{\circ}$.

These conclusions are consistent with results from VLBI and hydrodynamical simulations (Mooley et al. 2018a), from parametric modeling of the jet (Ghirlanda et al. 2018; Hotokezaka et al. 2018; Troja et al. 2018c), and from polarization (Corsi et al. 2018). The jet opening angle for GW170817 appears to be somewhat smaller than the median found for $\mathrm{SHBs},\left\langle\theta_{j}\right\rangle \simeq 16^{\circ} \pm 10^{\circ}\left(\theta_{j}\right.$ estimates for bursts displaying jet breaks lie between $\sim 2^{\circ}-8^{\circ}$; e.g., Fong et al. 2015), but is consistent with the estimates for SHBs like 090510 and 150101B (Kumar \& Barniol Duran 2010; Nicuesa Guelbenzu et al. 2012; Troja et al. 2018b, see also Troja et al. 2018c) and the tail end of the long GRB distribution (e.g., Goldstein et al. 2016). With the confirmation of the successful jet in GW170817, our polarization upper limit from Corsi et al. (2018) implies high isotropy of the magnetic field.

At early times ( $\simeq 100$ days), the steady rise in the light curve indicated that there was continuous energy injection within the outflow emitting in our line of sight (e.g., Nakar \& Piran 2018), and implied the presence of a mildly relativistic wide-angle outflow with significant angular and/or radial structure (consistent with a cocoon-dominated outflow). At later times, the successful narrow jet came into view and dominated the late-time afterglow. The confirmation of a jet strengthens the link between neutron star mergers and regular SHBs.

Like some previous long-duration GRBs (e.g., Berger et al. 2003), GW170817 has given us insights into the structure of the wide-angle outflows surrounding the jet core, and the simple top-hat jet for SHBs will likely have to be revised (Lazzati et al. 2017a, 2017b; Nakar \& Piran 2017). The confirmation of the wide angle outflow that dominated the outflow at early times bodes well for the future detection of the EM counterparts of GW sources observed at larger viewing angles.

The National Radio Astronomy Observatory is a facility of the National Science Foundation operated under cooperative agreement by Associated Universities, Inc. The Australia Telescope is funded by the Commonwealth of Australia for operation as a National Facility managed by CSIRO. We thank the staff of the GMRT that made these observations possible. GMRT is run by the National Centre for Radio Astrophysics of the Tata Institute of Fundamental Research. The MeerKAT telescope is operated by the South African Radio Astronomy Observatory, (SARAO), which is a facility of the National Research Foundation (NRF), an agency of the Department of Science and Technology. The authors thank the NRAO staff, especially Mark Claussen and Amy Mioduszewski, for scheduling the VLA observations. K.P.M. would like to thank Kenta Hotokezaka and Michael Zhang for help with emcee. We thank Varun Bhalerao for help with some of the uGMRT observation A.C. acknowledges support from the NSF CAREER award \#1455090. D.D. is supported by an Australian Government Research Training Program Scholarship. K.P.M. is currently a Jansky Fellow of the National Radio Astronomy Observatory. T.M. acknowledges the support of the
Australian Research Council through grant FT150100099. Part of this work was supported by the GROWTH (Global Relay of Observatories Watching Transients Happen) project funded by the National Science Foundation under PIRE grant No. 1545949. DK was additionally supported by by NSF grant AST-1412421. P.C. acknowledges support from the Department of Science and Technology via Swarana Jayanti Fellowship award (file no.DST/SJF/PSA-01/2014-15). The research of O.S. is supported by the South African Research Chairs Initiative of the Department of Science and Technology and NRF.

Software: Matplotlib (Hunter 2007), NumPy (Oliphant 2006), MIRIAD (Sault et al. 1995), DIFMAP (Shepherd 1997), CASA (McMullin et al. 2007), emcee (Foreman-Mackey et al. 2013), corner (Foreman-Mackey 2016).

\section{ORCID iDs}

K. P. Mooley (ib https://orcid.org/0000-0002-2557-5180

D. Dobie (i) https://orcid.org/0000-0003-0699-7019

E. Lenc (i) https://orcid.org/0000-0002-9994-1593

A. Corsi (i) https://orcid.org/0000-0001-8104-3536

T. Murphy (i) https://orcid.org/0000-0002-2686-438X

D. L. Kaplan (i) https://orcid.org/0000-0001-6295-2881

G. Hallinan (iD https://orcid.org/0000-0002-7083-4049

F. Camilo (i) https://orcid.org/0000-0002-1873-3718

M. M. Kasliwal (iD https://orcid.org/0000-0002-5619-4938

S. R. Kulkarni (i) https://orcid.org/0000-0001-5390-8563

P. A. Woudt (iD https://orcid.org/0000-0002-6896-1655

\section{References}

Abbott, B. P., Abbott, R., Abbott, T. D., et al. 2017a, PhRvL, 119, 161101 Abbott, B. P., Abbott, R., Abbott, T. D., et al. 2017b, ApJL, 848, L12 Alexander, K. D., Berger, E., Fong, W., et al. 2017, ApJL, 848, L21 Alexander, K. D., Margutti, R., Blanchard, P. K., et al. 2018, ApJL, 863, L18 Arcavi, I., Hosseinzadeh, G., Howell, D. A., et al. 2017, Natur, 551, 64 Berger, E., Kulkarni, S. R., Pooley, G., et al. 2003, Natur, 426, 154 Beuermann, K., Hessman, F. V., Reinsch, K., et al. 1999, A\&A, 352, L26 Camilo, F., Scholz, P., Serylak, M., et al. 2018, ApJ, 856, 180

Corsi, A., Hallinan, G. W., Lazzati, D., et al. 2018, ApJL, 861, L10 Coulter, D. A., Foley, R. J., Kilpatrick, C. D., et al. 2017, Sci, 358, 1556 Cowperthwaite, P. S., Berger, E., Villar, V. A., et al. 2017, ApJL, 848, L17 D’Avanzo, P., Campana, S., Salafia, O. S., et al. 2018, A\&A, 613, L1 Dobie, D., Kaplan, D. L., Murphy, T., et al. 2018, ApJL, 858, L15 Drout, M. R., Piro, A. L., Shappee, B. J., et al. 2017, Sci, 358, 1570 Duffell, P. C., Quataert, E., Kasen, D., \& Klion, H. 2018, arXiv:1806.10616 Evans, P. A., Cenko, S. B., Kennea, J. A., et al. 2017, Sci, 358, 1565 Fong, W., Berger, E., Margutti, R., \& Zauderer, B. A. 2015, ApJ, 815, 102 Foreman-Mackey, D. 2016, JOSS, 1, 24

Foreman-Mackey, D., Hogg, D. W., Lang, D., \& Goodman, J. 2013, PASP, 125,306

Frail, D. A., Soderberg, A. M., Kulkarni, S. R., et al. 2005, ApJ, 619, 994 Frail, D. A., Waxman, E., \& Kulkarni, S. R. 2000, ApJ, 537, 191

Galama, T. J., Wijers, R. A. M. J., Bremer, M., et al. 1998, ApJL, 500, L97 Ghirlanda, G., Salafia, O. S., Paragi, Z., et al. 2018, arXiv:1808.00469 Gill, R., \& Granot, J. 2018, MNRAS, 478, 4128

Goldstein, A., Connaughton, V., Briggs, M. S., \& Burns, E. 2016, ApJ, 818, 18 Goldstein, A., Veres, P., Burns, E., et al. 2017, ApJL, 848, L14 Gottlieb, O., Nakar, E., \& Piran, T. 2018, MNRAS, 473, 576 Granot, J., \& Kumar, P. 2003, ApJ, 591, 1086

Haggard, D., Nynka, M., Ruan, J. J., et al. 2017, ApJL, 848, L25 Hallinan, G., Corsi, A., Mooley, K. P., et al. 2017, Sci, 358, 1579 Harrison, F. A., Bloom, J. S., Frail, D. A., et al. 1999, ApJL, 523, L121 Hotokezaka, K., Nakar, E., Gottlieb, O., et al. 2018, arXiv:1806.10596 Hunter, J. D. 2007, CSE, 9, 90

Jonas, J. \& MeerKAT Team 2016, in Proc. MeerKAT Science: On the Pathway to the SKA, https://pos.sissa.it/cgi-bin/reader/conf.cgi?confid=277</ A $>$,id. 1,1 
Kasen, D., Metzger, B., Barnes, J., Quataert, E., \& Ramirez-Ruiz, E. 2017, Natur, 551, 80

Kasliwal, M. M., Nakar, E., Singer, L. P., et al. 2017, Sci, 358, 1559

Kathirgamaraju, A., Barniol Duran, R., \& Giannios, D. 2018, MNRAS, 473, L121

Kumar, P., \& Barniol Duran, R. 2010, MNRAS, 409, 226

Kumar, P., \& Granot, J. 2003, ApJ, 591, 1075

Lamb, G. P., Mandel, I., \& Resmi, L. 2018, MNRAS, 481, 2581

Lazzati, D., Deich, A., Morsony, B. J., \& Workman, J. C. 2017a, MNRAS, 471, 1652

Lazzati, D., López-Cámara, D., Cantiello, M., et al. 2017b, ApJL, 848, L6

Lazzati, D., Perna, R., Morsony, B. J., et al. 2018, PhRvL, 120, 241103

Lipunov, V. M., Gorbovskoy, E., Kornilov, V. G., et al. 2017, ApJL, 850, L1

Livio, M., \& Waxman, E. 2000, ApJ, 538, 187

Lyman, J. D., Lamb, G. P., Levan, A. J., et al. 2018, NatAs, 2, 751

Margutti, R., Alexander, K. D., Xie, X., et al. 2018, ApJL, 856, L18

Margutti, R., Berger, E., Fong, W., et al. 2017, ApJL, 848, L20

Matsumoto, T., Nakar, E., \& Piran, T. 2018, arXiv:1807.04756

McMullin, J. P., Waters, B., Schiebel, D., Young, W., \& Golap, K. 2007, in ASP Conf. Ser. 376, Astronomical Data Analysis Software and Systems XVI, ed. R. A. Shaw, F. Hill, \& D. J. Bell (San Francisco, CA: ASP), 127

Mészáros, P., Rees, M. J., \& Wijers, R. A. M. J. 1998, ApJ, 499, 301

Mooley, K. P., Deller, A. T., Gottlieb, O., et al. 2018a, Natur, 561, 355

Mooley, K. P., Nakar, E., Hotokezaka, K., et al. 2018b, Natur, 554, 207

Nakar, E., Gottlieb, O., Piran, T., Kasliwal, M. M., \& Hallinan, G. 2018, arXiv: 1803.07595

Nakar, E., \& Piran, T. 2017, ApJ, 834, 28

Nakar, E., \& Piran, T. 2018, MNRAS, 478, 407

Nicuesa Guelbenzu, A., Klose, S., Krühler, T., et al. 2012, A\&A, 538, L7

Nynka, M., Ruan, J. J., Haggard, D., \& Evans, P. A. 2018, ApJL, 862, L19
Oliphant, T. E. 2006, A Guide to NumPy (USA: Trelgol Publishing)

Panaitescu, A., \& Kumar, P. 2003, ApJ, 592, 390

Pian, E., D’Avanzo, P., Benetti, S., et al. 2017, Natur, 551, 67

Reddy, S. H., Kudale, S., Gokhale, U., et al. 2017, JAI, 6, 1641011

Resmi, L., Schulze, S., Ishwara Chandra, C. H., et al. 2018, ApJ, submitted (arXiv:1803.02768)

Rossi, E., Lazzati, D., \& Rees, M. J. 2002, MNRAS, 332, 945

Ruan, J. J., Nynka, M., Haggard, D., Kalogera, V., \& Evans, P. 2018, ApJL, 853, L4

Sari, R., Piran, T., \& Halpern, J. P. 1999, ApJL, 519, L17

Sari, R., Piran, T., \& Narayan, R. 1998, ApJL, 497, L17

Sault, R. J., Teuben, P. J., \& Wright, M. C. H. 1995, in ASP Conference Series, Vol. 77, Astronomical Data Analysis Software and Systems IV, ed. R. A. Shaw, H. E. Payne, \& J. J. E. Hayes (San Francisco, CA: ASP), 433 Shepherd, M. C. 1997, in ASP Conf. Ser. 125, Astronomical Data Analysis Software and Systems VI, ed. G. Hunt \& H. Payne (San Francisco, CA: ASP), 77

Smartt, S. J., Chen, T.-W., Jerkstrand, A., et al. 2017, Natur, 551, 75

Soares-Santos, M., Holz, D. E., Annis, J., et al. 2017, ApJL, 848, L16

Soderberg, A. M., Kulkarni, S. R., Nakar, E., et al. 2006, Natur, 442, 1014

Tanvir, N. R., Levan, A. J., González-Fernández, C., et al. 2017, ApJL, 848, L27

Troja, E., Piro, L., Ryan, G., et al. 2018a, MNRAS, 478, L18

Troja, E., Piro, L., van Eerten, H., et al. 2017, Natur, 551, 71

Troja, E., Ryan, G., Piro, L., et al. 2018b, arXiv:1806.10624

Troja, E., van Eerten, E. T. H., Ryan, G., et al. 2018c, arXiv:1808.06617

Valenti, S., Sand, D. J., Sheng, Y., et al. 2017, ApJL, 848, L24

van der Horst, A. J., Kamble, A., Resmi, L., et al. 2008, A\&A, 480, 35

van Eerten, H., van der Horst, A., \& MacFadyen, A. 2012, ApJ, 749, 44

Xie, X., Zrake, J., \& MacFadyen, A. 2018, ApJ, 863, 58 